\title{
The Cotangent Bundle of a Cominuscule Grassmannian
}

\author{
V. Lakshmibai, Vijay Ravikumar, \& William Slofstra
}

\begin{abstract}
A theorem of the first author states that the cotangent bundle of the type $A$ Grassmannian variety can be embedded as an open subset of a smooth Schubert variety in a two-step affine partial flag variety. We extend this result to cotangent bundles of cominuscule generalized Grassmannians of arbitrary Lie type.
\end{abstract}

\section{Introduction}

An earlier work of Lusztig and Strickland suggests possible connections between the conormal varieties to partial flag varieties on the one hand and affine Schubert varieties on the other. In particular, Lusztig [8] relates certain orbit closures arising from the type $A$ cyclic quiver $\hat{A}_{h}$ to affine Schubert varieties. In the case $h=2$, Strickland [11] relates such orbit closures to conormal varieties of determinantal varieties; furthermore, any determinantal variety can be canonically realized as an open subset of a Schubert variety in the Grassmannian [6].

Inspired by these results, the first author was interested in finding a relationship between affine Schubert varieties and conormal varieties to the Grassmannian. As a first step, she showed that the compactification of the cotangent bundle to the Grassmannian is canonically isomorphic to a Schubert variety in a two-step affine partial flag variety [5]. In this paper, we extend her result to cominuscule generalized Grassmannians of arbitrary finite type (such Grassmannians occur in types $A-E$ ).

\subsection{Preliminaries}

Let $G_{0}$ be a simple algebraic group over $\mathbb{C}$ with associated Lie algebra $\mathfrak{g}_{0}$ and simple roots $\left\{\alpha_{1}, \ldots, \alpha_{n}\right\}$. A simple root $\alpha_{i}$ is cominuscule if the coefficient of $\alpha_{i}$ in any positive root of $\mathfrak{g}_{0}$ (written in the simple root basis) is less than or equal to 1 .

The Weyl group of $G_{0}$ is generated by simple reflections $S_{0}:=\left\{s_{1}, \ldots, s_{n}\right\}$ corresponding to the simple roots $\left\{\alpha_{1}, \ldots, \alpha_{n}\right\}$. For any subset $K \subset S_{0}$, we let $P_{K} \subset G_{0}$ denote the parabolic subgroup whose Weyl group is generated by the elements of $K$. For $1 \leq i \leq n$, set $S_{0, i}:=S_{0} \backslash\left\{s_{i}\right\}$, so that $P_{S_{0, i}}$ is a maximal parabolic subgroup of $G_{0}$. The manifold $G_{0} / P_{S_{0, i}}$ is called a generalized Grassmannian of type $G_{0}$ and is said to be cominuscule if $\alpha_{i}$ is cominuscule. For additional background on cominuscule Grassmannians, see [1] or [7].

Received May 30, 2015. Revision received August 21, 2016. 
Table 1 Finite type Dynkin diagrams with cominuscule simple root marked in black (left column), and the corresponding affine Dynkin diagrams with both the cominuscule and the additional affine root marked in black (right column)

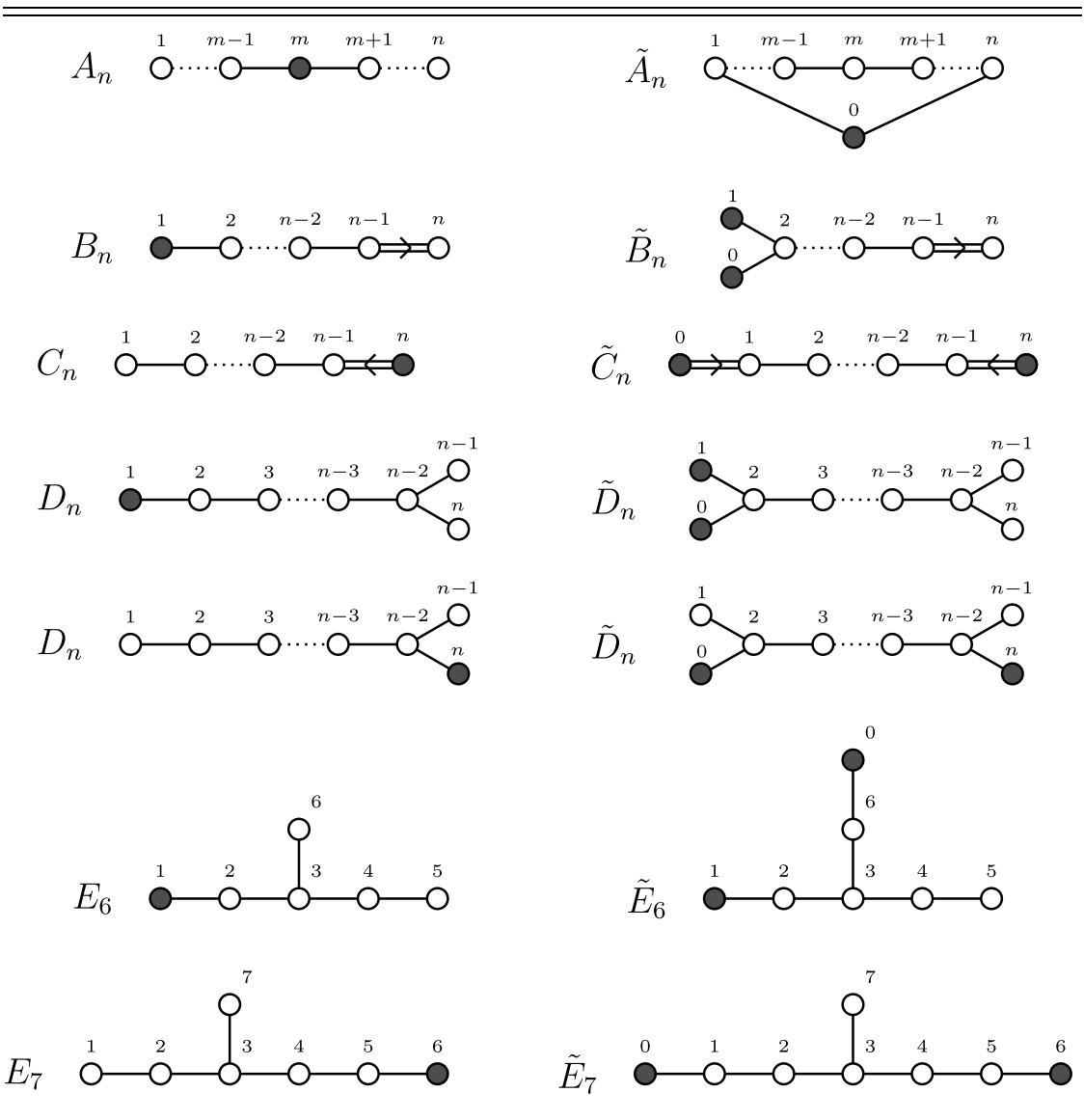

For the remainder of the paper, we fix $m \in[1, n]$ and consider the generalized Grassmannian $X:=G_{0} / P_{S_{0, m}}$ associated with $\alpha_{m}$. Note that $\alpha_{m}$ is not yet assumed to be cominuscule.

Let $\mathfrak{g}$ denote the affine untwisted Kac-Moody algebra associated to $\mathfrak{g}_{0}$, and let $\mathcal{G}$ be the corresponding affine Kac-Moody group (see $[4, \S 6]) .{ }^{1}$ The Dynkin diagram for $\mathfrak{g}$ depends on the Dynkin diagram for $\mathfrak{g}_{0}$ and is shown in Table 1 (see $[2, \S 18.1]$ or $[3, \S 4.8]$ ). We use the convention that the affine node (sometimes called the special node) is labeled by zero, and similarly let $\alpha_{0}$ and $s_{0}$ be the affine

\footnotetext{
${ }^{1}$ We use the calligraphic font (e.g., $\mathcal{G}$ and $\mathcal{P}_{J}$ ) for infinite-dimensional Kac-Moody groups and a noncalligraphic font for finite-dimensional Lie groups (e.g., $G_{0}, P_{J}$ ).
} 
simple root and reflection, respectively. The Weyl group $W$ of $\mathfrak{g}$ is generated by $S:=\left\{s_{0}, \ldots, s_{n}\right\}$, and there is a parahoric subgroup $\mathcal{P}_{K} \subset \mathcal{G}$ associated with any subset $K \subset S$. We let $\mathcal{X}_{K}:=\mathcal{G} / \mathcal{P}_{K}$ denote the associated affine flag variety, and $W_{K} \subset W$ denote the Weyl group of $\mathcal{P}_{K}$ or, in other words, the subgroup of $W$ generated by $K$. For any subsets $I \subset K \subset S$, let $W_{K}^{I} \subset W_{K}$ denote the set of minimal length coset representatives of $W_{K} / W_{I}$. In particular, $W^{K}:=W_{S}^{K}$ is the set of minimal length coset representatives of $W / W_{K}$, and elements $w \in W_{K}$ index Schubert varieties $\mathcal{X}_{K}(w)$ of $\mathcal{X}_{K}$.

Observe that $S_{0}=S \backslash\left\{s_{0}\right\}$. Let $S_{m}:=S \backslash\left\{s_{m}\right\}$ and $J:=S_{0, m}=S \backslash\left\{s_{0}, s_{m}\right\}$. Let $w_{i}$ be the maximal element of $W_{S_{i}}^{J}$, where $i \in\{0, m\}$. It is a standard fact that $\mathcal{X}_{S_{m}}\left(w_{0}\right) \cong \mathcal{X}_{J}\left(w_{0}\right) \cong X$ (see Lemma 2.3). The basis of this note is the following elementary but crucial observation.

Lemma 1.1. If $\alpha_{m}$ is cominuscule in $\mathfrak{g}_{0}$, then $\mathcal{X}_{J}\left(w_{0}\right)$ and $\mathcal{X}_{J}\left(w_{m}\right)$ are isomorphic.

Proof. The list of cominuscule simple roots in each type is well known. We indicate the cominuscule simple roots for each Dynkin diagram (up to diagram automorphism) in the left column of Table 1 and the corresponding untwisted affine Dynkin diagram in the right column. In each case, the Dynkin diagram of $W_{S_{0}}$ is isomorphic to the Dynkin diagram of $W_{S_{m}}$, and this isomorphism identifies $\alpha_{m}$ with the affine root $\alpha_{0}$. Consequently, $\mathcal{X}_{J}\left(w_{0}\right)$ and $\mathcal{X}_{J}\left(w_{m}\right)$ are isomorphic.

\subsection{Main Results}

Consider the Schubert variety $Y:=\mathcal{X}_{J}\left(w_{0} w_{m}\right)$ in $\mathcal{X}_{J}$. The Kac-Moody group $\mathcal{G}$ acts on $\mathcal{X}_{J}$ by left multiplication, and since $G_{0}$ is the Levi subgroup of $\mathcal{P}_{S_{0}} \subset \mathcal{G}$, we can regard $Y$ as a $G_{0}$-variety.

In fact, $Y$ can naturally be considered as a $G_{0}$-homogeneous fibre bundle over $X$. More precisely:

THEOREM 1.2. The affine Schubert variety $Y=\mathcal{X}_{J}\left(w_{0} w_{m}\right)$ is stable under the left action of $G_{0} \subset \mathcal{G}$, and the natural projection $Y \rightarrow \mathcal{X}_{S_{m}}\left(w_{0}\right) \cong X$ is a $G_{0^{-}}$ homogeneous fiber bundle map with fiber $\mathcal{X}_{J}\left(w_{m}\right)$. In particular, $Y$ is smooth.

Our main result is that if $X$ is cominuscule, then $Y$ is a natural compactification of the cotangent bundle $T^{*} X$ :

Theorem 1.3. If $X$ is cominuscule, then the fiber $\mathcal{X}_{J}\left(w_{m}\right)$ is isomorphic to $X$, and there is a $G_{0}$-equivariant map $\tilde{\mu}: T^{*} X \rightarrow Y$ of fiber bundles over $X$ under which $T^{*} X$ is isomorphic to a dense open subset of $Y$.

We prove Theorem 1.2 in Section 2 and Theorem 1.3 in Section 3. In order to prove Theorem 1.3 , we explicitly construct the $G_{0}$-equivariant embedding $\tilde{\mu}$ : $T^{*} X \rightarrow Y$ that maps the base $X$ isomorphically onto the Schubert variety $\mathcal{X}_{J}\left(w_{0}\right)$ and maps the fiber over the identity to a dense open subset of the Schubert variety $\mathcal{X}_{J}\left(w_{m}\right)$. 
When $X$ is minuscule rather than cominuscule, it is natural to replace $\mathcal{G}$ with a twisted affine Kac-Moody group. Theorem 1.2 still holds in this case, but as we show in Section 4, Theorem 1.3 does not hold. In this case, the variety $Y$ is not the compactification of the cotangent bundle $T^{*} X$, but of a different bundle over $X$.

We note that Manivel and Michalek [9] have recently studied the local geometry of tangential varieties (which are compactifications of the tangent bundle) to cominuscule Grassmannians. We do not know if there is a version of our compactification for the tangent bundle.

\section{The Fiber Bundle Structure on $Y$}

We note that $\alpha_{m}$ is not assumed to be cominuscule in this section. Given $I \subset K \subset$ $S$, we can write any $w \in W^{I}$ uniquely as $w=v u$, where $v \in W^{K}$ and $u \in W_{K}^{I}$. In this case, the projection $\mathcal{G} / \mathcal{P}_{I} \rightarrow \mathcal{G} / \mathcal{P}_{K}$ induces a projection $\mathcal{X}_{I}(w) \rightarrow \mathcal{X}_{K}(v)$, and the generic fiber of this projection is $\mathcal{X}_{I}(u)$. We say that $w=v u$ is a parabolic decomposition with respect to $K$.

For any $v \in W$, we define $\operatorname{Supp}(v):=\{s \in S \mid s \leq v\}$ to be the set of simple reflections contained in a reduced expression for $v$. For any $u \in W$, let $D^{I}(u):=$ $\left\{s \in S \mid s u \leq_{I} u\right\}$, where $\leq_{I}$ is the Bruhat order on $W / W_{I}$. We have the following proposition from [10, Thm. 2.3 and Prop. 3.2].

Proposition 2.1. The projection $\mathcal{X}_{I}(w) \rightarrow \mathcal{X}_{K}(v)$ is a fiber bundle with fiber $\mathcal{X}_{I}(u)$ if and only if $\operatorname{Supp}(v) \cap K \subset D^{I}(u)$.

When the condition $\operatorname{Supp}(v) \cap K \subset D^{I}(u)$ is satisfied, we say that $w=v u$ is a Billey-Postnikov decomposition with respect to $K$.

Recall that for any $s \in S$, we have $s w \leq_{I} w$ if and only if $\mathcal{X}_{I}(w)$ is stable under left multiplication by the rank 1 parahoric subgroup $\mathcal{P}_{\{s\}}$. It follows that if $L=D^{I}(w)$, then $\mathcal{X}_{I}(w)$ is stable under the action of the parahoric subgroup $\mathcal{P}_{L}$ ([1], see also [10, Lemma 3.9]).

LeMMA 2.2. Let $y=w_{0} w_{m}$, so $Y=\mathcal{X}_{J}(y)$.

(a) $y=w_{0} w_{m}$ is a Billey-Postnikov decomposition with respect to $S_{m}$.

(b) $D^{J}(y)=S_{0}$.

Proof. Since $w_{i}$ is maximal in $W_{S_{i}}^{J}$, we know that $D^{J}\left(w_{i}\right)=S_{i}$ for $i \in\{0, m\}$. It is clear that $w_{0} w_{m}$ is a parabolic decomposition with respect to $S_{m}$ and $\operatorname{Supp}\left(w_{0}\right) \cap$ $S_{m}=S_{0} \cap S_{m}=J \subset D^{J}\left(w_{m}\right)$, proving part (a).

For part (b), if $z \in W_{S_{0}}$, then $z w_{0} \leq_{J} w_{0}$, and hence $z w_{0}=v_{0} z^{\prime}$, where $v_{0} \in$ $W_{S_{0}}^{J}$ and $z^{\prime} \in W_{J}$. Similarly, $z^{\prime} w_{m}=v_{m} z^{\prime \prime}$, where $v_{m} \in W_{S_{m}}^{J}$ and $z^{\prime \prime} \in W_{J}$. So $z y=v_{0} v_{m} z^{\prime \prime} \leq_{J} y$, and hence $S_{0} \subset D^{J}(y)$. But $D^{J}(y)$ must be a proper subset of $S$, so $D^{J}(y)=S_{0}$. 
Given $K \subseteq S$, the Levi subgroup $G_{K}$ of $\mathcal{P}_{K}$ is a Kac-Moody group with Weyl group $W_{K}$. Since $\mathcal{G}$ is affine, if $K$ is a strict subset of $S$, then $G_{K}$ is finitedimensional, and similarly $W_{K}$ is finite. In order to prove Theorem 1.2, we need the following standard lemma.

Lemma 2.3. If $K, I \subsetneq S$ and $w \in W_{K}^{K \cap I}$, then $P_{K, I}:=G_{K} \cap \mathcal{P}_{I}$ is the parabolic subgroup of $G_{K}$ corresponding to the subgroup $W_{K \cap I} \subseteq W_{K}$, and $\mathcal{X}_{I}(w)$ is isomorphic to a Schubert variety in the flag variety $G_{K} / P_{K, I}$. In particular, if $w$ is the maximal element of $W_{K}^{K \cap I}$, then $\mathcal{X}_{I}(w)$ is isomorphic to $G_{K} / P_{K, I}$.

Now the proof of Theorem 1.2 follows immediately from Lemma 2.2.

Proof of Theorem 1.2. By part (b) of Lemma 2.2 the variety $Y=\mathcal{X}_{J}\left(w_{0} w_{m}\right)$ is stable under the left action of $G_{0}$. The base $\mathcal{X}_{S_{m}}\left(w_{0}\right)$ is clearly $G_{0}$-stable as well, and the natural projection $Y \rightarrow \mathcal{X}_{S_{m}}\left(w_{0}\right)$ is $G_{0}$-equivariant. By part (a) of Lemma 2.2 and Proposition 2.1 the projection $Y \rightarrow \mathcal{X}_{S_{m}}\left(w_{0}\right)$ is a $G_{0^{-}}$ homogeneous fiber bundle with fiber $\mathcal{X}_{J}\left(w_{m}\right)$.

Now the Levi subgroup $G_{S_{0}}$ of $\mathcal{P}_{S_{0}}$ is simply $G_{0}$. Since $S_{m} \cap S_{0}=J$ and $w_{0}$ is the maximal element of $W_{S_{0}}^{J}$, we can alternately set $I=S_{m}$ in Lemma 2.3 to get $\mathcal{X}_{S_{m}}\left(w_{0}\right) \cong \mathcal{X}_{J}\left(w_{0}\right) \cong X=G_{0} / P_{J}$. Similarly, $\mathcal{X}_{J}\left(w_{m}\right)$ is isomorphic to the flag variety $G_{S_{m}} / P_{J}$. Since $Y$ is a fiber bundle with smooth fiber and base, it follows that $Y$ is smooth.

\section{The Cotangent Bundle}

If $X$ is cominuscule, then $\mathcal{X}_{J}\left(w_{m}\right)$ is isomorphic to $X$ by Lemma 1.1. To prove Theorem 1.3, we explicitly construct the map $T^{*} X \hookrightarrow Y$. Let $\mathcal{B}$ be the Borel subgroup of the Kac-Moody group $\mathcal{G}$ (in the literature, $\mathcal{B}$ is also known as the Iwahori subgroup of $\mathcal{G}$ ). For convenience, we write $G_{i}$ for the Levi subgroup $G_{S_{i}}$ of the parahoric subgroup $\mathcal{P}_{S_{i}} \subset \mathcal{G}$, where $i \in\{0, m\}$ (in particular, $G_{0}$ is the same as before). We let $B_{i}:=G_{i} \cap \mathcal{B}$ be the induced Borel of $G_{i}$, and $P_{i}:=B_{i} W_{J} B_{i}=$ $G_{i} \cap \mathcal{P}_{J}$. Finally, let $U_{i} \subset P_{i}$ be the unipotent radical of $P_{i}$. As in the previous section, $P_{0}=P_{J}, X=G_{0} / P_{0}$, and moreover $\mathcal{X}_{J}\left(w_{i}\right) \cong G_{i} / P_{i}$ for $i \in\{0, m\}$. Note that $P_{0}$ depends on $\alpha_{m}$ as well as $\alpha_{0}$.

We will also need to use the underlying Lie algebras. We assume the standard construction of $\mathfrak{g}$, in which

$$
\mathfrak{g} \cong \mathfrak{g}_{0} \otimes_{\mathbb{C}} \mathbb{C}\left[z, z^{-1}\right] \oplus \mathbb{C} c \oplus \mathbb{C} d
$$

as a vector space (see $[2, \S 18.1]$ or $[3, \S 7.2])$. Let $\mathfrak{h}$ be the Cartan subalgebra of the Kac-Moody algebra $\mathfrak{g}$. Let $\mathfrak{g}_{i} \subset \mathfrak{g}$ be the (finite-dimensional) Lie algebra of $G_{i}$, where $i \in\{0, m\}$. Let $\mathfrak{u}_{i} \subset \mathfrak{g}_{i}$ be the (nilpotent) Lie algebra of $U_{i}$. Finally, let $\mathfrak{u}_{m}^{-}$be the opposite nilpotent radical to $\mathfrak{u}_{m}$ inside $\mathfrak{g}_{m}$. We consider the linear map

$$
\phi: \mathfrak{u}_{0} \rightarrow \mathfrak{g} \text { defined by } x \mapsto x \otimes z^{-1} .
$$

In order to prove Theorem 1.3, we need the following lemma. 
Lemma 3.1. The map $\phi: \mathfrak{u}_{0} \rightarrow \mathfrak{u}_{m}^{-}$is a $P_{0}$-equivariant isomorphism of vector spaces.

Proof. Let $R$ denote the set of roots of $\mathfrak{g}$, with simple roots $\Delta:=\left\{\alpha_{0}, \ldots, \alpha_{n}\right\}$. The simple roots of $\mathfrak{g}_{0}$ and $\mathfrak{g}_{m}$ are the subsets of $\Delta$ obtained by omitting $\alpha_{0}$ and $\alpha_{m}$, respectively. For any subalgebra $\mathfrak{a} \subset \mathfrak{g}$, we let $R(\mathfrak{a})$ denote the set of $\mathfrak{h}$ weights of $\mathfrak{a}$, and let $R^{+}(\mathfrak{a})$ and $R^{-}(\mathfrak{a})$ denote the subsets of positive and negative roots, respectively. Let $\theta$ be the highest root of $\mathfrak{g}_{0}$, and let $\delta=\alpha_{0}+\theta$ be the basic imaginary root of $\mathfrak{g}([2, \S 17.1]$ or $[3, \S 5.6])$.

We can describe the set of roots of $\mathfrak{g}$ by

$$
R(\mathfrak{g})=\left\{\alpha+k \delta \mid \alpha \in R\left(\mathfrak{g}_{0}\right), k \in \mathbb{Z}\right\} \cup\left\{k \delta \mid k \in \mathbb{Z}_{\neq 0}\right\} .
$$

The set of positive roots of $\mathfrak{g}$ is given by

$$
R^{+}(\mathfrak{g})=R^{+}\left(\mathfrak{g}_{0}\right) \cup\left\{\alpha+k \delta \in R \mid \alpha \in R\left(\mathfrak{g}_{0}\right), k \in \mathbb{Z}_{>0}\right\} \cup\left\{k \delta \mid k \in \mathbb{Z}_{>0}\right\} .
$$

Note that $R\left(\mathfrak{u}_{0}\right) \subset R^{+}\left(\mathfrak{g}_{0}\right)$ and $R\left(\mathfrak{u}_{m}\right) \subset R^{+}\left(\mathfrak{g}_{m}\right)$. Using the simple roots of $\mathfrak{g}_{0}$ and $\mathfrak{g}_{m}$, the roots of $\mathfrak{u}_{0}$ and $\mathfrak{u}_{m}$ can then be written

$$
\begin{aligned}
R\left(\mathfrak{u}_{0}\right) & =\left\{\sum_{i=1}^{n} a_{i} \alpha_{i} \in R^{+}(\mathfrak{g}) \mid a_{m}=1\right\} \text { and } \\
R\left(\mathfrak{u}_{m}\right) & =\left\{a_{0} \alpha_{0}+\sum_{i \in[1, n] \backslash\{m\}} a_{i} \alpha_{i} \in R^{+}(\mathfrak{g}) \mid a_{0}=1\right\},
\end{aligned}
$$

where the requirement that $a_{m}=1$ (resp. $a_{0}=1$ ) follows from the fact that $\alpha_{m}$ is cominuscule in $\mathfrak{g}_{0}$ (resp. $\alpha_{0}$ is cominuscule in $\mathfrak{g}_{m}$ ).

Every root of $\mathfrak{g}_{0}$ can be written uniquely as $\theta-\sum_{i=1}^{n} a_{i} \alpha_{i}$, where $a_{i} \geq 0$ for all $1 \leq i \leq n$. Since $\alpha_{m}$ is cominuscule, the coefficient of $\alpha_{m}$ in $\theta$ is equal to 1 . Using the previous description of $R\left(\mathfrak{u}_{0}\right)$, it follows that $\alpha \in R\left(\mathfrak{g}_{0}\right)$ is an element of $R\left(\mathfrak{u}_{0}\right)$ if and only if

$$
\alpha=\theta-\sum_{i \in[1, n] \backslash\{m\}} a_{i} \alpha_{i}
$$

for some coefficients $a_{i} \geq 0$ (in particular, note that any $\alpha$ of this form cannot belong to $R^{-}\left(\mathfrak{g}_{0}\right)$ since it will have a positive $\alpha_{m}$-coefficient).

Note that for any $\alpha \in R\left(\mathfrak{u}_{0}\right)$, the homomorphism $\phi$ maps $\mathfrak{g}_{\alpha}$ isomorphically onto $\mathfrak{g}_{\alpha-\delta}$. Thus, the $\mathfrak{h}$-weights of $\phi\left(\mathfrak{u}_{0}\right)$ are precisely

$$
\begin{aligned}
& \left\{\alpha-\delta \in R \mid \alpha=\theta-\sum_{i \in[1, n] \backslash\{m\}} a_{i} \alpha_{i}, \text { where } a_{i} \geq 0 \text { for } i \in[1, n] \backslash\{m\}\right\} \\
& =\left\{-(-\theta+\delta)-\sum_{i \in[1, n] \backslash\{m\}} a_{i} \alpha_{i} \in R^{-} \mid a_{i} \geq 0 \text { for all } i \in[1, n] \backslash\{m\}\right\} .
\end{aligned}
$$

This latter set is exactly the negative of the $\mathfrak{h}$-weights of $\mathfrak{u}_{m}$ since $(-\theta+\delta)=\alpha_{0}$ and since $\alpha_{0}$ is cominuscule in $\mathfrak{g}_{m}$ as in Lemma 1.1. We conclude that $\phi\left(\mathfrak{u}_{0}\right)=$ $\mathfrak{u}_{m}^{-}$. Since $\phi$ is a clearly bijective, it is a vector space isomorphism. 
Consider the left adjoint action of $\mathfrak{p}_{0}:=\operatorname{Lie}\left(P_{0}\right)$ on $\mathfrak{g}$. Under this action, each element of the weight space $\mathfrak{g}_{\beta} \subset \mathfrak{p}_{0}$ maps $\mathfrak{g}_{\alpha}$ into $\mathfrak{g}_{\alpha+\beta}$ whenever $\alpha+\beta \in$ $R(\mathfrak{g})$ and annihilates $\mathfrak{g}_{\alpha}$ otherwise. Recall that $R\left(\mathfrak{p}_{0}\right)=R^{+}\left(\mathfrak{g}_{0}\right) \cup\left\{\sum_{i=1}^{n} a_{i} \alpha_{i} \in\right.$ $\left.R^{-}\left(\mathfrak{g}_{0}\right) \mid a_{m}=0\right\}$ and observe that both $\mathfrak{u}_{0}$ and $\mathfrak{u}_{m}^{-}$are stable under the left adjoint action of $\mathfrak{p}_{0}$ and, moreover, that $\phi$ is $\mathfrak{p}_{0}$-equivariant. It follows that $\phi$ is $P_{0}$-equivariant.

Using the map $\phi: \mathfrak{u}_{0} \rightarrow \mathfrak{u}_{m}^{-}$, we construct a map

$$
\Phi: \mathfrak{u}_{0} \rightarrow \mathcal{X}_{J}=\mathcal{G} / \mathcal{P}_{J}: x \mapsto\left[\exp (\phi(x)) \cdot \mathcal{P}_{J}\right]
$$

Lemma 3.2. $\Phi$ is a $P_{0}$-equivariant algebraic isomorphism from $\mathfrak{u}_{0}$ to an open dense subset of $\mathcal{X}_{J}\left(w_{m}\right)$.

Proof. The exponential map $\mathfrak{u}_{m}^{-} \rightarrow \exp \left(\mathfrak{u}_{m}^{-}\right)=: U_{m}^{-}$is an algebraic isomorphism, and $U_{m}^{-} \cong U_{m}^{-} \cdot\left[e \mathcal{P}_{J}\right]$ is an open dense subset of $\mathcal{X}_{J}\left(w_{m}\right)=G_{m} / P_{m}$, where $e \in$ $\mathcal{G}$ is the identity. Since $\phi$ is a $P_{0}$-equivariant bijection and $P_{0} \subset \mathcal{P}_{J}$, the result follows.

We can now finish the proof of the main theorem.

Proof of Theorem 1.3. As in Section 2, we let $Y=\mathcal{X}_{J}(y)$, where $y=w_{0} w_{m}$. The cotangent bundle of $X$ is

$$
T^{*} X=G_{0} \times P_{0} \mathfrak{u}_{0},
$$

the quotient of $G_{0} \times \mathfrak{u}_{0}$ by the $P_{0}$-action $p \cdot(g, x)=\left(g p, p^{-1} x\right)$. We can define a map

$$
\mu: G_{0} \times \mathfrak{u}_{0} \rightarrow \mathcal{X}_{J}(y):(g, x) \mapsto g \cdot \Phi(x),
$$

where we use the fact that $\Phi(x) \in \mathcal{X}_{J}\left(w_{m}\right) \subset \mathcal{X}_{J}(y)$, which is stable under the left action of $G_{0}$ by Theorem 1.2. But $\mu$ is $P_{0}$-equivariant, so we get an induced map

$$
\tilde{\mu}: G_{0} \times P_{0} \mathfrak{u}_{0} \rightarrow \mathcal{X}_{J}(y) .
$$

The cotangent bundle map $T^{*} X \rightarrow X$ sends $(g, x) \mapsto\left[g P_{0}\right]$. Since the projection $\mathcal{G} \mapsto \mathcal{G} / \mathcal{P}_{S_{m}}$ sends $g \cdot \Phi(x) \mapsto\left[g \mathcal{P}_{S_{m}}\right]$, we conclude that the diagram

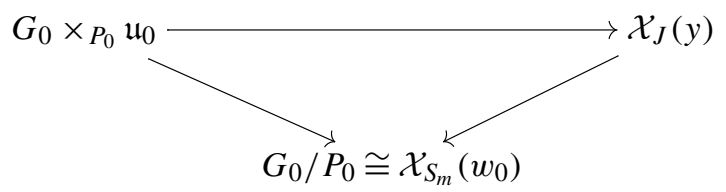

commutes, and thus $\tilde{\mu}$ is a morphism of $G_{0}$-homogeneous fibre bundles. Over $\left[e P_{0}\right]$, this map restricts to $\Phi: \mathfrak{u}_{0} \rightarrow \mathcal{X}_{J}\left(w_{m}\right)$, which is injective and has open dense image in the fiber $\mathcal{X}_{J}\left(w_{m}\right)$. We conclude that the total map $G_{0} \times{ }_{P_{0}} \mathfrak{u}_{0} \rightarrow$ $\mathcal{X}_{J}(y)$ is injective and has an open image. 
Table 2 Finite type Dynkin diagrams with minuscule simple root marked in black (left column), and the corresponding twisted affine Dynkin diagrams with both the minuscule and the additional affine root marked in black (right column). We use Kac's notation for the twisted affine Dynkin diagrams. Note that in Dynkin's notation, $A_{2 n-1}^{(2)}$ is denoted $\tilde{B}_{n}^{t}$, and $D_{n+1}^{(2)}$ is denoted $\tilde{C}_{n}^{t}$

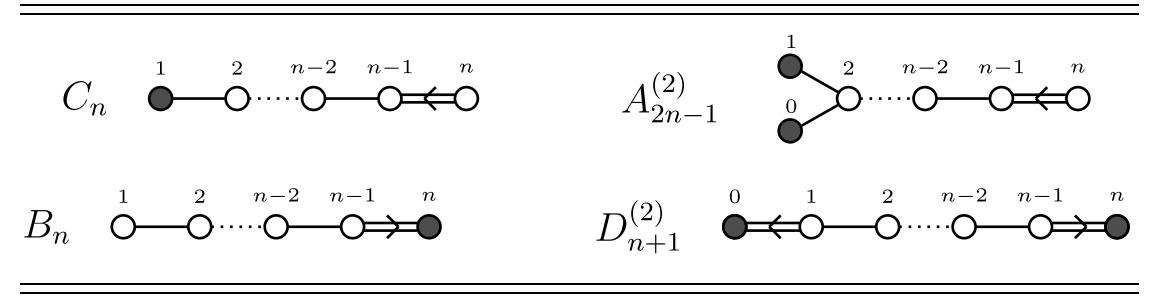

\section{Minuscule Grassmannians}

A Grassmannian $X=G_{0} / P_{S_{0, m}}$ is minuscule if $\alpha_{m}^{\vee}$ is cominuscule in the dual root system. The minuscule and cominuscule Grassmannians coincide in types $A, D$, and $E$ but are disjoint in the other types. There are just two families of Grassmannians that are minuscule but not cominuscule: $\mathrm{SO}(2 n+1) / P_{S_{0, n}}$, the Grassmannian corresponding to the root $\alpha_{n}$ in type $B_{n}$, and $\operatorname{Sp}(2 n) / P_{S_{0,1}}$, the Grassmannian corresponding to the root $\alpha_{1}$ in $C_{n}$. The corresponding Dynkin diagrams are listed in Table 2. As algebraic varieties, $\operatorname{Sp}(2 n) / P_{S_{0,1}}$ is isomorphic to $\mathbb{P}^{2 n-1}$, and $\mathrm{SO}(2 n+1) / P_{S_{0, n}}$ is isomorphic to $\mathrm{SO}(2 n+2) / P_{n} \cong \mathrm{SO}(2 n+2) / P_{n+1}$, so each minuscule Grassmannian is isomorphic to a cominuscule Grassmannian. However, the minuscule Grassmannians are distinct as homogeneous spaces, and their cotangent bundles are distinct as homogeneous bundles.

Suppose that $\alpha_{m}$ is minuscule but not cominuscule, and let $\mathfrak{g}$ and $\mathcal{G}$ be the affine twisted Kac-Moody algebra and group associated to $\mathfrak{g}_{0}$ (see [2, §18.4] or [3], and $[4, \S 6])$. The proof of Theorem 1.2 still works in this setting, and consequently the affine Schubert variety $Y=\mathcal{X}_{J}\left(w_{0} w_{1}\right) \subseteq \mathcal{X}_{J}:=\mathcal{G} / \mathcal{P}_{J}$ is a fiber bundle over $X$ with fiber $\mathcal{X}_{J}\left(w_{m}\right)$. Furthermore, following Lemma 1.1, we have $\mathcal{X}_{J}\left(w_{m}\right) \cong$ $\mathcal{X}_{J}\left(w_{0}\right) \cong X$ (see Table 2 for the proof).

With all these pieces in place, we might expect that $Y$ is a compactification of $T^{*} X$ as in the cominuscule case. However, the argument from the cominuscule setting breaks down at this point. Specifically, the argument from Section 3 shows that $Y$ is a compactification of the homogeneous vector bundle $T:=G_{0} \times P_{0} \mathfrak{u}_{m}^{-}$ on $X$. However, $T$ is not the cotangent bundle of $X$. Indeed, by the following lemma, $T$ splits as the direct sum of two $G_{0}$-homogeneous vector bundles on $X$, whereas $T^{*} X$ does not.

Lemma 4.1. As $P_{0}$-modules, $\mathfrak{u}_{m}^{-}$splits as the direct sum of two submodules, whereas $\mathfrak{u}_{0}$ does not. 
Proof. Let $\delta=\alpha_{0}+\theta_{0}$ be the basic imaginary root of $\mathfrak{g}$, where $\theta_{0}$ is the highest short root of $\mathfrak{g}_{0}([2, \S 17.1]$ or $[3, \S 8.3])$. For any subalgebra $\mathfrak{a} \subset \mathfrak{g}$, let $R_{S}(\mathfrak{a})$ (resp. $R_{l}(\mathfrak{a})$ ) denote the set of real short (resp. long) $\mathfrak{h}$-weights of $\mathfrak{a}$ (see [2, §17.2] or $[3, \S 5.1])$. The set of roots of $\mathfrak{g}$ is given by

$$
\left\{\alpha+k \delta: \alpha \in R_{S}\left(\mathfrak{g}_{0}\right), k \in \mathbb{Z}\right\} \cup\left\{\alpha+2 k \delta: \alpha \in R_{l}\left(\mathfrak{g}_{0}\right), k \in \mathbb{Z}\right\} \cup\left\{k \delta: k \in \mathbb{Z}_{\neq 0}\right\} .
$$

Moreover, the $\mathfrak{h}$-weights of $\mathfrak{u}_{0}$ and $\mathfrak{u}_{m}^{-}$are given by

$$
\begin{aligned}
R\left(\mathfrak{u}_{0}\right) & =\left\{\sum_{i \in[1, n]} a_{i} \alpha_{i} \in R^{+}(\mathfrak{g}): a_{m} \in\{1,2\}\right\} \text { and } \\
R\left(\mathfrak{u}_{m}^{-}\right) & =\left\{a_{0} \alpha_{0}+\sum_{i \in[1, n] \backslash\{m\}} a_{i} \alpha_{i} \in R^{-}(\mathfrak{g}): a_{0} \in\{-1,-2\}\right\} .
\end{aligned}
$$

Write $\mathfrak{u}_{m}^{-}=\mathfrak{u}_{m, s}^{-} \oplus \mathfrak{u}_{m, l}^{-}$, where $\mathfrak{u}_{m, s}^{-}:=\bigoplus_{\alpha \in R_{s}\left(\mathfrak{u}_{m}^{-}\right)} \mathfrak{g}_{\alpha}$ and $\mathfrak{u}_{m, l}^{-}:=\bigoplus_{\alpha \in R_{l}\left(\mathfrak{u}_{m}^{-}\right)} \mathfrak{g}_{\alpha}$. The short (resp. long) $\mathfrak{h}$-weights of $\mathfrak{u}_{m}^{-}$are precisely those with $\alpha_{0}$ coefficient $a_{0}=-1$ (resp. $a_{0}=-2$ ) in the simple root basis. It follows that the left adjoint action of $P_{0}$ preserves the long and short roots of $u_{m}^{-}$, and hence $T=G_{0} \times P_{0}$ $u_{m, s}^{-} \oplus G_{0} \times P_{0} u_{m, l}^{-}$is a direct sum of two homogeneous vector bundles. On the other hand, $\mathfrak{u}_{0}$ does not split as a $P_{0}$-module since the Lie algebra $\mathfrak{p}_{0}$ of $P_{0}$ can take short roots of $\mathfrak{u}_{0}$ (which have $\alpha_{m}$ coefficient 1) to long roots (which have $\alpha_{m}$ coefficient 2).

Let $\mathfrak{h}_{0}$ and $H_{0}$ denote the Cartan subalgebra and subgroup of $\mathfrak{g}_{0}$ and $G_{0}$, respectively. An $H_{0}$-module $M$ is attractive if there is some $\omega$ in $\mathfrak{h}_{0}$ such that $\alpha(\omega)>0$ for all $\mathfrak{h}_{0}$-weights $\alpha$ of $M$. The fact that $Y$ cannot be the compactification of $T^{*} X$ follows from the following more general result.

Lemma 4.2. Given $P_{0}$-modules $U$ and $V$, suppose that there exists an element $\omega \in$ $\mathfrak{h}_{0}$ with the property that $\alpha(\omega)>0$ for any $\mathfrak{h}_{0}$-weight $\alpha$ of $U$ or $V$. Furthermore, if both $G_{0} \times{ }_{P_{0}} U$ and $G_{0} \times{ }_{P_{0}} V$ embed as open dense homogeneous $G_{0}$-bundles in a homogeneous $G_{0}$-fiber bundle $Y$, then $U$ and $V$ are isomorphic as $P_{0}$-modules.

Proof. We can think of $U$ and $V$ as open dense subsets of the fiber over the identity in $Y$. As such, the intersection of $U$ and $V$ is nonempty. Let $y$ be a point of the intersection. Since $\alpha(\omega)>0$ for all $\mathfrak{h}_{0}$-weights $\alpha$ of $U$ or $V$, the limit

$$
\lim _{n \rightarrow \infty} \exp (-n \omega) \cdot y
$$

exists and is equal to both $0_{U}$ and $0_{V}$, the zero elements of $U$ and $V$, which in particular must be equal. The sets $U$ and $V$ are both open, and $0:=0_{U}=0_{V}$ is a $P_{0}$-fixed point in both $U$ and $V$, so $U \cong T_{0} U=T_{0} V \cong V$ as $P_{0}$-modules.

Corollary 4.3. There is no open embedding of $T^{*} X$ into $Y$ as $G_{0}$-homogeneous fiber bundles over $X$.

Proof. Suppose on the contrary that such an embedding exists. Note that $\alpha\left(\omega_{m}\right)>$ 0 for all $\alpha \in R\left(\mathfrak{u}_{0}\right)$, where $\omega_{m} \in \mathfrak{h}_{0}$ is the fundamental weight dual to the simple 
root $\alpha_{m}$. Moreover, the roots of $\mathfrak{u}_{m}^{-}$are all of the form $\alpha-\delta$ or $\alpha-2 \delta$ with $\alpha \in$ $R\left(\mathfrak{u}_{0}\right)$. Since $\delta\left(\omega_{m}\right)=0$, it follows that $\beta\left(\omega_{m}\right)>0$ for all $\beta \in R\left(\mathfrak{u}_{m}^{-}\right)$(indeed, $\mathfrak{u}_{0}$ and $\mathfrak{u}_{m}^{-}$have the same $\mathfrak{h}_{0}$-weights since $\delta(\omega)=0$ for any $\left.\omega \in \mathfrak{h}_{0}\right)$. By Lemma 4.2 it follows that $\mathfrak{u}_{0}$ and $\mathfrak{u}_{m}^{-}$are isomorphic as $P_{0}$-modules, contradicting Lemma 4.1.

AcKnOwLEDGments. The second author thanks K. N. Raghavan for useful discussions. The Dynkin diagrams in Tables 1 and 2 are based on the excellent TikZ templates due to Oscar Castillo-Felisola. The first author was supported by NSA Grant H98230-11-1-0197. The second author was partially supported by a fellowship from the Infosys Foundation. The authors are grateful to the referees for their useful comments.

\section{References}

[1] S. Billey and V. Lakshmibai, Singular loci of Schubert varieties, Birkhäuser Boston, Boston, 2000.

[2] R. Carter, Lie algebras of finite and affine type, Cambridge University Press, Cambridge, 2005.

[3] V. Kac, Infinite dimensional Lie algebras, third edition, Cambridge University Press, Cambridge, 1990.

[4] S. Kumar, Kac-Moody groups, their flag varieties and representation theory, Birkhäuser Boston, Boston, 2002.

[5] V. Lakshmibai, Cotangent bundle to the Grassmann variety, Transform. Groups 21 (2016), no. 2, 519-530.

[6] V. Lakshmibai and C. S. Seshadri, Geometry of $G / P-I I$, Proc. Indiana Acad. Sci. 87A (1978), 1-54.

[7] J. M. Landsberg and L. Manivel, On the projective geometry of rational homogeneous varieties, Comment. Math. Helv. 78 (2003), 65-100.

[8] G. Lusztig, Canonical bases arising from quantized enveloping algebras, J. Amer. Math. Soc. 3 (1990), 447-498.

[9] L. Manivel and M. Michałek, Secants of minuscule and cominuscule minimal orbits, Linear Algebra Appl. 481 (2015), 288-312.

[10] E. Richmond and W. Slofstra, Billey-Postnikov decompositions and the fibre bundle structure of Schubert varieties, Math. Ann. 366 (2016), no. 1, 31-55.

[11] E. Strickland, On the conormal bundle of the determinantal variety, J. Algebra 75 (1982), 523-537.

V. Lakshmibai

Department of Mathematics

College of Arts and Sciences

Northeastern University

567 Lake Hall

Boston, Massachusetts, 02115

USA
V. Ravikumar

Chennai Mathematical Institute

H1, SIPCOT IT Park

Siruseri, Tamilnadu, 603103

India

vijayr@cmi.ac.in

\section{lakshmibai@neu.edu}


W. Slofstra

Institute for Quantum Computing

University of Waterloo

200 University Ave. West

Waterloo, Ontario, N2L 3G1

Canada

weslofst@uwaterloo.ca 